\title{
Physiological Adaptation And Transcriptome Changes In The Plateau Plant Common Vetch (Vicia Sativa L.) In Response To The Plain Environment
}

Jinglei He

Northeast Agricultural University

Lingzelai Kong

Northeast Agricultural University

Jinqiu $Y u$

Northeast Agricultural University

Bing Li

Northeast Agricultural University

XiangYin Hou

Northeast Agricultural University

Yuying Yuan

Northeast Agricultural University

Hang Yin

Northeast Agricultural University

Guowen Cui ( $\nabla$ cgw603@163.com )

Northeast Agricultural University

\section{Research Article}

Keywords: Qinghai-Tibet Plateau, plain, physiological, transcriptome.

Posted Date: September 24th, 2021

DOl: https://doi.org/10.21203/rs.3.rs-191828/v2

License: (c) (1) This work is licensed under a Creative Commons Attribution 4.0 International License.

Read Full License 


\section{Abstract}

Common vetch (Vicia sativa L.) is an annual herb with high nutritional value, strong adaptability and cold tolerance. It is one of the forage varieties widely planted in the construction of artificial grassland in Qinghai Tibet Plateau. In order to reveal the molecular regulation mechanism of common vetch introduced into plain, physiological and transcriptome analysis of common vetch seedlings in Plateau and plain environment were carried out. In the plain environment, the leaf structure and some physiological indexes of common vetch can adapt to the plain environment gradually and keep stable. However, the maximum photochemical quantum yield ( $\mathrm{fv} / \mathrm{fm}$ ) and chlorophyll content (Chl) of PSII fluctuated and could not keep stable. Further transcriptome sequencing showed that there were many different genes involved in photosynthesis pathway, photosynthetic antenna protein pathway, carbon dioxide fixation pathway in photosynthetic organisms and porphyrin and chlorophyll metabolism pathway in plain environment. Similarly, TF analysis showed that MYB, NAC, AP2-EREBP and Orphans were the main transcription factors involved in the adaptation of common vetch to the changes of plain environment. These results may explain that the main reason why the common vetch is not suitable for the plain environment is the difference of the light intensity between the plain and the plateau. These findings provide a theoretical basis for scientific introduction and breeding of new varieties from plateau to plain.

\section{Background}

The Qinghai-Tibet Plateau, known as the "third pole of the world" and the "Asian water tower", has an average altitude of more than 4000 meters and contains vast alpine grasslands ${ }^{1}$. Its characteristics include low temperatures, low oxygen levels, poor soils and strong ultraviolet radiation. High solar radiation and its effects on secondary metabolites of higher plants in high-altitude areas have been confirmed in many studies ${ }^{2}$. In the past 20 years, due to the influence of global climate change, the rate of increase in temperature on the plateau has been much faster than that in most low-lying areas of the world ${ }^{3-5}$, and the temperature in the dry period is the limiting factor of vegetation growth in Tibet ${ }^{6}$. The phenology of alpine grasslands has been a hot topic in the scientific community, especially the adaptive genetic mechanism of nonmodel organisms that can be studied in the whole genome by high-throughput sequencing technology ${ }^{7-8}$.

Plateau plants have genes that are positively selected, such as those involved in the response to radiation, DNA repair, membrane stability and organic metabolism. These functions may be the reason why plants adapt to high radiation, low temperature and soil depletion environments ${ }^{9}$. When the light needed for photosynthesis is suddenly reduced, light harvesting complex II (LHCII) can maintain the redox balance between the photosystem I (PSI) and photosystem II (PSII) ${ }^{10}$. The cytochrome (CYP) of different plants may be a positive or negative regulator of drought tolerance. A study on the plateau plant Sophora psammophila showed that drought stress increased the transcription of CYP and that CYP may be an active regulator of drought tolerance ${ }^{11}$. The largest cold-inducible transcription factor (TF) group in 
Elymus nutans is the AP2/EREBP family. In addition, MADS, Sigma70-like, and Alfin-like also have different expression patterns ${ }^{12}$.

Common vetch (Vicia sativa L.) is an annual legume that is widely distributed in the Mediterranean basin, West Asia, Central Asia and China. Because of its strong cold resistance and high seed yield, it is an important economic feed variety for providing absorbable crude protein to domestic animals in highaltitude areas ${ }^{13}$. Research shows that an increase in temperature during the growing season will affect the seed yield and quality of Astragalus sinicus, thus affecting its utilization ${ }^{14}$.

With the increasing demand for animal protein, the demand for animal feed rich in protein is also increasing ${ }^{15}$. However, due to the aggravation of land degradation and the impact of climate change and other factors on the mainland, the original feed crops have been unable to meet the needs of humans. Increasing the basic sources of animal feed and improving utilization efficiency will be of great significance to the development of sustainable animal husbandry ${ }^{16}$. To study the adaptability and regulation mechanism of plateau plants to the plain environment, seedlings of common vetch were transported from the plateau ecosystem to the plain ecosystem. The main objectives of this study are (1) to observe the changes in the morphological structure and leaf structure of plants in the plain environment; (2) to explore the response time of common vetch to adapt to the plain environment through the measurement of physiological indexes; and (3) to identify the different genes involved in the response time to adapt to the plain environment and identify the key genes related to growth and development by combining the results of transcriptome analyses. Then, our goal is to identify the genetic changes in plateau-adapted plants as they grow in plain areas. This information can be used to provide technical support for the introduction of common vetch in plain areas.

\section{Methods}

\section{Plant materials}

The experiment was carried out in a field in Sangzhuzi District, Tibet Autonomous Region in 2019. Seeds of common vetch (Vicia sativa L.), variety XiMu324, obtained from the Qinghai Academy of Animal Husbandry and Veterinary Sciences, were planted in a round plastic basin (diameter: $10 \mathrm{~cm}$ and height: $15 \mathrm{~cm}$ ). The soil was a uniform nutrient soil. The plants were transported in the basin during the seedling stage to the outdoor experimental site of Northeast Agricultural University of Harbin City by air (Additional file 1: Table S1). Samples were collected on the first, third, fifth and seventh days (J1, J3, J5, and J7, respectively). The samples collected before high-altitude transportation were set as the control (J0). After sampling from 9:00 to $11: 00$, the plants were frozen in liquid nitrogen and stored in a refrigerator at $-80^{\circ} \mathrm{C}$, and three independent samples were collected daily for physiological index measurements. The samples collected on the 3rd and 7th days were used for transcriptome sequencing.

\section{Measurement of stomatal density and leaf structure}


Fresh leaves were cut into $2 \times 5 \mathrm{~mm}$ strips and stored in glutaraldehyde. The leaves were dehydrated with a series of solutions. The stomatal opening and closing of leaves were observed with an S-3400N (Hitachi, China) scanning electron microscope. Five field images were saved for each sample, and the number of stomata was calculated.

Veinless common vetch leaves were fixed in FAA solution to prepare paraffin patches $(3 \times 5 \mathrm{~mm})$ and stained with solid green and safranin. Cross-sections of $10 \mu \mathrm{m}$ thick leaves were analyzed under a 10fold microscope (Nikon Eclipse 50i, Japan) to observe the status of the palisade tissue and spongy tissue.

\section{Measurement of chlorophyll fluorescence and physiological index}

Chlorophyll fluorescence ( $\mathrm{Fv} / \mathrm{Fm})$ was measured by a portable photosynthetic apparatus after 30 minutes of leaf shading. After the samples were transported back to the laboratory, physiological indexes, namely, the osmotic regulation system (soluble sugar, soluble protein), antioxidant system (SOD, POD, CAT), oxidation system $\left(\mathrm{O}_{2}^{-}, \mathrm{H}_{2} \mathrm{O}_{2}\right)$ and MDA, were determined with a test kit (Comin, Suzhou, China).

\section{RNA extraction and library preparation for transcriptome sequencing}

Total RNA was isolated from common vetch by using a NEBNext ${ }^{\circledR}$ Ultra ${ }^{\text {TM }}$ RNA Library Prep Kit (NEB, USA). Agarose gel electrophoresis ( $1 \%$ gel concentration) and a NanoPhotometer ${ }^{\circledR}$ spectrophotometer (IMPLEN, CA, USA) were used to detect whether the RNA was degraded and the RNA purity. The cDNA library was prepared with a NEBNext ${ }^{\circledR}$ Ultra ${ }^{\text {TM }}$ RNA Library Prep Kit for the Illumina sequencing system (NEB, USA). The mRNA was isolated and purified from the total RNA by magnetic beads linked with polyT oligosaccharides. The first cDNA was synthesized from the interrupted mRNA, and the second-strand cDNA was then synthesized by DNA polymerase I and RNase H. An Ampure XP system (Beckman Coulter, Beverly, USA) was used to select the fragment size (250-300 bp), and PCR was used to enrich the cDNA library. The quality of the constructed library was evaluated by an Agilent Bioanalyzer 2100 system. Finally, the Illumina HiSeq platform was used to perform high-throughput sequencing. The transcriptome datasets can be accessed in the NCBI Sequences Read Archive under accession number PRJNA688111.

\section{Functional annotation and differentially expressed gene (DEG) analysis}

The FASTX toolkit was used to read the clean sequences. The Trinity program was used for the assembly of single genes. The NR, NT (http://www.ncbi.nlm.nih.gov/), PFAM (http://pfam.sanger.ac.uk/), GO (http://www.geneontology.org/), and KOG (http://www.ncbi.nlm.nih.gov/COG/) databases were used to annotate gene functions. The differential expression analysis of the two samples was performed using the DEGseq (2010) R package. P values were adjusted using the q value. Q value $<0.005 \& \| \log _{2}$ Qfold change区| $>1$ was set as the threshold for significantly differential expression. All the DEGs were subjected to enrichment analysis according to GO functions and KEGG pathways. 


\section{Quantitative real-time PCR (qRT-PCR) analysis}

To confirm the DEG results, 8 transcripts were randomly selected from the photosynthesis pathways (ko00195) and carbon fixation in photosynthetic organisms pathways (ko00710) for qRT-PCR verification. Primer 5 software was used to design gene-specific qRT-PCR primers (Attached file 1: table S2).

A LightCycler Multiplex DNA Master Kit (CWBIO, China) was used to perform qRT-PCR quantitative analysis. The $10 \mu \mathrm{L}$ reaction mixture was composed of $5 \mu \mathrm{L}$ of $5 \mathrm{X}$ reaction mixture, $3.6 \mu \mathrm{L}$ of distilled water, $1 \mu \mathrm{L}$ of cDNA and $0.4 \mu \mathrm{L}$ of each primer $(10 \mathrm{mM})$. qRT-PCR analysis was performed on a LightCycler 480 real-time PCR system (Roche, Switzerland) $\left(95^{\circ} \mathrm{C}\right.$ for 3 minutes, 40 cycles of $95^{\circ} \mathrm{C}$ for 10 seconds, $58^{\circ} \mathrm{C}$ for 30 seconds, and $72^{\circ} \mathrm{C}$ for 30 seconds). The $2^{-\Delta \Delta} \mathrm{CT}$ calculation method was used to analyze the gene expression data.

\section{Statistical analysis}

SPSS 19.0 software was used to perform one-way analysis of variance (ANOVA) to evaluate the difference between the plateau condition and the control treatment. ANOVA with Duncan's comparison was considered to show significant differences at the $5 \%$ level in all comparisons. At least three biological replicates of the controls and samples were used for each statistical analysis. Microsoft Excel 2016 was used for data analysis, and SigmaPlot was used to draw the graphs.

\section{Results}

\section{Changes in the number of stomata and in the anatomical structure of leaves}

Compared with the control, the stomatal opening number of common vetch leaves decreased significantly on the first day, $50 \%$ lower than that of the control. It increased significantly on the third day, and reached a relatively stable state on the fifth and seventh days. On the 7th day, the highest number of stomata was 34.5 , which was $44.9 \%$ higher than that of the control (Fig. 1). The leaves of common vetch are double-sided, and the mesophyll tissue is divided into palisade tissue and spongy tissue. On the first day after transportation to the plain, the arrangement of upper epidermis cells, lower epidermis cells, palisade cells and sponge cells was loose, and the intercellular space was larger than that of the control. From the third day, the intercellular space and spongy tissue gradually recovered (Fig. 2).

\section{Changes in chlorophyll content and chlorophyll fluorescence}

The maximum photochemical quantum yield $(\mathrm{fv} / \mathrm{fm})$ of PSII reflects the potential maximum photosynthetic capacity of plants. Under non stress conditions, the change of this parameter is very small, and it is not affected by species and growth conditions. Compared with the first day, $\mathrm{fv} / \mathrm{fm}$ content increased on the third day. It was significantly higher on the 5th day than on the 3rd day, but significantly lower on the 7th day than that of the control. Fv/fm content reached the maximum on the 5th day, 
increased by $6.6 \%$ compared with the control (Fig. 3a). Chlorophyll is the main pigment of photosynthesis and one of the main indexes to evaluate the photosynthetic capacity of leaves. The chlorophyll content of common vetch increased significantly on the first day and the fifth day after being transported from plateau to plain. Although it decreased significantly to the control level on the 3rd day, it was still significantly lower than the control level on the 7th day and lower than the control level on the 5th day (Fig. 3b).

\section{Physiological response of common vetch to the plain environment}

Malonic acid (MDA) accumulation is usually used to reflect the degree of membrane lipid peroxidation, which is a common basis to identify plant cell damage. As shown in Figure 3-4, compared with the control, the MDA content of common vetch was significantly decreased on the first day after being transported back to the plain. From the third day, it was significantly higher than the control level in plateau area. On the first day, the content of MDA was the lowest, $30.7 \%$ lower than that of the plateau control. On the 7th day, the content of MDA was the highest, increased by $27 \%$ compared with the plateau control. (Fig. 4a).

Compared with the control, the content of $\mathrm{H}_{2} \mathrm{O}_{2}$ gradually increased from the first day. The content of $\mathrm{H}_{2} \mathrm{O}_{2}$ was higher on the 5th day. On the 7th day and the 5th day, the content was the same, reaching a relatively stable state (Fig. 4b). Compared with the control, $\mathrm{O}_{2}{ }^{-}$content gradually increased in the first 5 days, but the change was not significant. Compared with the 5th day, the content of $\mathrm{O}_{2}{ }^{-}$decreased significantly on the 7th day, but it was still higher than the control level (Fig. 4c).

The accumulation of osmolytes has the function of scavenging reactive oxygen species and ensuring the structure of plant cell membrane. Soluble sugar content was significantly lower than the control level on the first day, and then increased significantly on the third day. On the first day, the soluble sugar content was the lowest, decreased by $33.5 \%$ compared with the control. On the 7th day, the soluble sugar content remained the same as that of the control (Fig. 4d). Compared with the control, the soluble protein content decreased significantly on the 1 st and 3rd day. Although the content increased significantly on the 5th day compared with the 3rd day, it decreased significantly on the 7th day and gradually returned to the level on the 3rd day. On the third day, the content of soluble protein decreased by $32.9 \%$ (Fig. 4e).

Antioxidant system can keep the balance of production and elimination of reactive oxygen species. Compared with the control, SOD activity decreased by $5.0 \%$ on the first day. Compared with the first day, it increased significantly on the third day. It decreased significantly on the 5th day and then increased significantly on the 7th day. The content of SOD activity was higher than that of the control except the first day, and reached the maximum on the seventh day (Fig. 4f). GSH activity was $26.0 \%$ lower than the control level on the first day. However, it increased significantly on the third day, but did not change significantly on the fifth and seventh days, and returned to the same activity level as the control (Fig. $4 \mathrm{~g})$.

\section{RNA sequencing and de novo assembly}


After the seedlings were transported to the plain, the photosynthetic pigment changed significantly on the third day and gradually recovered to stability on the seventh day. Therefore, to obtain the gene expression profile under plain and plateau conditions, the transcriptomes of the leaves of the plateau control samples and plain samples at 3 and 7 days were analyzed via an Illumina platform. From de novo assembly, a total of 129,209 transcripts and 38,702 unigenes were obtained with an average length of $1552 \mathrm{bp}$. We recorded an N50 value of $2394 \mathrm{bp}$ (Table. 1). The unigene length ranged from 301 to 16,715 bp. Among the unigenes, 10973 (28.35\%) were more than 2000 bp in length, and there was no single gene that was shorter than $300 \mathrm{bp}$. A total of 8789 (22.71\%) genes were $300-500 \mathrm{bp}$ in length, 9585 (24.77\%) were $500-1000$ bp in length, and 9355 (24.17\%) were $1000-2000$ bp in length (Table. 2).

The raw reads from one Illumina RNA-seq library generated in this study are available from the BioProject at NCBI (https://www.ncbi. nlm.nih.gov/bioproject/) under accession number PRJNA688111.

Table 1 Illumina RNA-seq reads and de novo assembly statistics of common vetch.

\begin{tabular}{ll} 
Total number of raw reads & $247,896,340$ \\
\hline Total number of clean reads & $60,060,559$ \\
\hline Mean length of reads $(\mathrm{bp})$ & 1919 \\
\hline Number of total unigenes & 38,702 \\
\hline Mean length of unigenes (bp) & 1552 \\
\hline Minimum unigene length (bp) & 301 \\
\hline Maximum unigene length (bp) & 16,715 \\
\hline N50 (bp) & 2394
\end{tabular}

Table 2 Length distribution of the unigenes.

\begin{tabular}{|lllllll|}
\hline Length (nt) & Total & $<300$ & $300-500$ & $500-1000$ & $1000-2000$ & $\geq 2000$ \\
\hline Number of Unigenes & 38702 & 0 & 8789 & 9585 & 9355 & 10973 \\
\hline$\%$ & $100 \%$ & $0 \%$ & $22.71 \%$ & $24.77 \%$ & $24.17 \%$ & $28.35 \%$ \\
\hline
\end{tabular}

\section{Functional annotation}

Among the 38,702 unigenes, a total of 29,384 unigenes were annotated in at least one database, including 25,225 unigenes annotated in the NR database, 25,201 unigenes in NT, 18,771 unigenes in PFAM, 18,771 unigenes in GO, and 6042 unigenes in the KOG database (Fig. 5a).

Compared with the annotation results from the NR database, it can be seen that the most homologous species with common vetch is Medicago truncatula, with 8391 homology (33.3\%). In addition, $18.4 \%$ of unigenes were similar to those of Trifolium pratense (Additional file 1: Figure S3.). In accordance with GO 
analysis, 105146 single genes were classified according to the three major GO categories. There were 25 groups of biological processes (BPs), 20 groups of cellular components (CCs) and 10 groups of molecular functions (MFs) (Fig. 5b, additional file 4: Table S3). The most abundant G0 terms in the unigenes of common vetch were "metabolic process" (10131), "cellular process" (11068) and "singleorganism process" (8056) for BP; "cell part" (6127) and "cell" (6127) for CC; and "catalytic activity" (8350) and "binding" $(10,690)$ for MF.

After KEGG annotation, 8185 DEGs were mapped into 130 KEGG metabolic pathways. The top three KEGG pathways were "metabolism" (4870), "genetic information processing" (2099) and "environmental information processing" (412). The pathways with the highest proportion of genes to the total number of annotated genes were ribosome (ko03010, 510 single genes), plant pathogen interaction (ko04626, 304 single genes), carbon metabolism (ko01200, 3011 single genes) and plant hormone signal transduction (ko04075, 274 single genes) (Fig. 5c, Additional file 5: Table S4.).

\section{Overall analysis of DEGs}

In this experiment, we used deseq software to analyze the differential expression among the sample groups, with FDR $<0.05$. Compared with the control (J0), samples collected on the third day (J3) had 3251 upregulated genes and 3317 downregulated genes, and 1359 upregulated genes and 1648 downregulated genes were observed on the seventh day (Fig. 6a). A total of 443 genes showed the same change in three comparisons when comparing the DEGs on different days. Compared with the control (J0), there were 1491 DEGs on the 3rd and 7th days (Fig. 6b). To confirm this result, 8 genes with different transcription abundances were verified by real-time RT-PCR. The gene expression levels obtained with the two methods were consistent.

In the $\mathrm{J} 3$ vs $\mathrm{J} 0$ comparison, the seven most enriched KEGG pathways are shown in Fig. 7a (Additional file 6: Table S5; Q value < 0.01). There were 28 DEGs in photosynthesis (ko00195), 15 in photosynthetic antenna protein (ko00196), 25 in photosynthetic biological carbon fixation (ko00710), 16 in phenylalanine metabolism (ko00360), 15 in porphyrin and chlorophyll metabolism (ko00860), and 14 in tyrosine metabolism (ko00350).

In the $\mathrm{J} 3$ vs $\mathrm{J} 0$ comparison, the four most enriched KEGG pathways are shown in Fig. 7b (Additional file 7: Table S6; Q value < 0.01). There were 206 DEGs in ribosomes (ko03010), 36 in photosynthesis (ko00195), 43 in ribosome biogenesis in eukaryotes (ko03008), and 21 in photosynthesis-antenna proteins (ko00196).

The pathways photosynthesis (ko00195) and photosynthesis-antenna proteins (ko00196) were identified in the $\mathrm{J} 3$ vs $\mathrm{J} 0$ and $\mathrm{J} 7$ vs $\mathrm{J} 0$ comparisons. The pathways identified in only the $\mathrm{J} 3$ vs $\mathrm{J} 0$ comparison were carbon fixation in photosynthetic organisms (ko00710), phenylalanine metabolism (ko00360), porphyrin and chlorophyll metabolism (ko00860), and tyrosine metabolism (ko00350). The pathways identified specifically in $\mathrm{J} 7 \mathrm{vs} \mathrm{J} 0$ were ribosome (ko03010) and ribosome biogenesis in eukaryotes (ko03008). 


\section{Differential expression of photosynthesis-related genes}

To study the adaptability of plateau plants to the plain environment, genes related to environmental effects were screened out from the filtered DEGs for further study. In J3 and J7, DEGs related to the reaction center of the PSI and PSII, photosynthetic antenna protein, electron transfer and $\mathrm{CO}_{2}$ fixation were identified. The main pathways were photosynthesis (k00195), photosynthesis-antenna proteins (ko00196) and photosynthesis biological carbon fixation (ko00710). When the plants were transported to the plain environment, the carbon fixation of photosynthetic organisms was inhibited. The main feature is that the core cytochrome $b_{6} / f(k 02636)$, which connects PSII and PSI, is downregulated during electron transfer. During photophosphorylation, the genes encoding FD-NADP ${ }^{+}$reductase (k02641) and ATP synthase (k02113, k02109) were downregulated (Fig. 8a).

During the Calvin cycle (Fig. 8b), the genes encoding Rubisco (k01601) in the pathway of 3phosphoglycerate (PGA) synthesis were downregulated. The genes encoding phosphoglycerate kinase (k00927) and glyceraldehyde-3-phosphate dehydrogenase (k05298) were downregulated in the synthesis of 3-phosphoglyceraldehyde (GAP). Many enzymes involved in the regeneration of ribulose-1,5disphosphate (RuBP) were downregulated. During the $\mathrm{C} 4$ cycle, oxaloacetic acid (OAA) is reduced to malic acid; the gene encoding malate dehydrogenase ( $\mathrm{k00025)}$ is downregulated. Regarding regeneration, the genes encoding pyruvate transaminase (k00814) were downregulated. However, the genes encoding aspartate transaminase ( $\mathrm{k} 14454)$, involved in transamination, were upregulated in the J3 vs J0 comparison. Lhca2, Lhca4, Lhcb1, Lhcb2, Lhcb3, Lhcb4, Lhcb5 and Lhcb6 in the photosynthetic antenna protein pathway, components of the complementary light complexes LCHI and LCHI, were downregulated. In addition, the genes encoding proteins in the reaction center of photosynthesis were downregulated, including PSI reaction center subunits III-VI (PsaF, PsaE, PsaG, PsaH), PSI reaction center subunits $\mathrm{X}$ and $\mathrm{XI}$ (PsaK, PsaL), PSI reaction center subunits PsaN and PsaO, and PSIl reaction center PsbK, PsbO, PsbQ, PsbW, and Psb27. In conclusion, the downregulation of these three pathways inhibited the growth of common vetch in the plain environment (Fig. 8c).

\section{Differential expression of encoding photosynthetic pigment-related genes}

The adaptability of plants to light is closely related to the photosynthetic pigment production pathway (Fig. 8d). In this study, the DEGs were mainly in the porphyrin and chlorophyll metabolism (ko0860) pathway. The genes encoding glutamine tRNA reductase (k02492), hydroxymethyldialane synthase (k01749), uroporphyrinogen decarboxylase (k01599), Mg-chelatase subunit $\mathrm{H}$ (k03403), magnesium-protoporphyrin 0-xymethyltransferase (k03428), magnesium-protoporphyrin IX monomethyl ester (oxidative) cyclase (k04035), protochlorophyllide reductase (k00218), chlorophyll An oxygenase (k13600) and chlorophyll a synthase (k04040) were downregulated in J3 compared with the control.

\section{Transcription factors (TFs) analysis}

Transcription factors (TFs), which widely exist in plants, play an important role in regulating plant growth and development and environmental stress response. In this study, 109 up-regulated TFs were identified 
in 39 gene families, mainly in NAC (11.01\%), Orphans (7.34\%) and HB (7.34\%) gene families. 94 down regulated TFs were distributed in 32 families, including MYB (15.96\%), AP2-EREBP (10.64\%) and Orphans (8.51\%) (Fig. 9a). Compared with J0, 206 transcription factors were identified in 52 gene families, mainly AP2-EREBP (12.62\%), mTERF (8.25\%), MYB (7.77\%) and Orphans (5.34\%). 238 down regulated TFs were distributed in 40 gene families, including MYB (13.45\%), AP2-EREBP (9.66\%) and NAC (8.40\%) (Fig. 9b). These results suggest that MYB, NAC, AP2-EREBP and orphans transcription factor family may play an important role in the adaptation of common vetch to plain environment.

\section{RNA-seq validation: qRT-PCR analysis}

We used qRT-PCR to detect the consistency of the expression patterns of 8 randomly selected genes. GAPA (Cluster-7705.13598, Cluster-7705.13381, Cluster-7705.13418), rbcS (Cluster-7705.13113), ATPF0B (Cluster-7705.13726), ATPF1D Cluster-7705.13438), petH (Cluster-7705.13612), and petC (Cluster7705.14151) were found to be downregulated in J3 vs J0 and J7 vs J0 by qRT-PCR. The qRT-PCR results for all genes were consistent with those of RNA-Seq, which provided strong support for the credibility of our RNA-seq data (Fig. 10).

\section{Discussion}

\section{Analysis of photosynthetic indexes of common vetch in response to environmental change}

Light is the direct energy source for plant growth. Light intensity is one of the most important factors that affect many physiological processes of plants, such as photosynthesis ${ }^{17,18}$. There is a threshold for the effect of light intensity on the growth of plants; light intensity that is too high or too low will inhibit the growth of plants ${ }^{19}$. In this experiment, the chlorophyll content of common vetch leaves changed significantly after transportation to the plain environment because of the high light intensity on the Qinghai-Tibet Plateau. On the 7th day, the chlorophyll content was significantly lower than that of the plateau control. This indicated that photosynthesis in the common vetch leaves was inhibited under lowlight conditions. When the light energy absorbed by plants exceeds the maximum light energy utilized by photosynthesis, the photosynthetic efficiency of plants may be reduced, resulting in the phenomenon of photoinhibition ${ }^{19}$. In this experiment, the chlorophyll content of common vetch increased significantly on the first day after it was transported to the plain. This may have occurred because the light intensity in the plain area was lower than that on the Qinghai-Tibet Plateau. However, under a suitable light intensity, common vetch produced more chlorophyll. The content of chlorophyll fluctuated over a few days. The difference in light intensity between the plateau and the plain may have been large enough to cause an obvious stress reaction after the common vetch was transported to the plain. Common vetch may have had difficulty in adapting to the plain environment within a short time. It is necessary to further analyze the reasons behind the change in photosynthesis when common vetch is transported to a plain environment. 
Changes in light intensity are also a main factor affecting leaf morphology. The plant leaf structure is very sensitive to changes in light intensity, and plants constantly adjust their leaf structure to adapt to different light intensity levels ${ }^{20}$. Higher chlorophyll content, higher stomatal density and thicker leaves are beneficial for increasing the photosynthetic rate. Under shading conditions, the stomatal density of soybean decreased significantly 21,22 . In this study, we found that when common vetch was stimulated by the environment on the first day (J1), the stomata of common vetch were closed, the intercellular space became larger, and the arrangement of upper and lower epidermis cells, palisade cells and sponge cells became loose due to the low light intensity. However, as the experimental period continued, the above indicators gradually returned to control levels. This result shows that plant leaves can recover from the influence of a sudden change in the altitudinal environment. The leaves of common vetch can adapt to changes altitude by adjusting their tissue structure.

\section{Analysis of physiological indexes of common vetch in response to environmental changes}

Some researchers have found that in changing light environments, plants can regulate their morphological and physiological states to maintain their normal life activities and simultaneously maintain the balance of their own internal systems. The morphological and physiological changes that occur in plants can increase their ability to obtain carbon from the environment ${ }^{23}$. Zhang ${ }^{24}$ suggested that the MDA content of alfalfa rhizomes increases with increasing low-light stress. In this study, the MDA content of common vetch was higher than that of the control except on the first day in the plain environment. The results showed that light intensity was the main reason for the significant increase in MDA content in common vetch in the plain environment. Jin ${ }^{25}$ showed that the soluble sugar content in plants decreased with increasing temperature. In this experiment, the soluble sugar content decreased significantly on the first day, then increased significantly, and finally returned to the control level. The content of soluble protein decreased throughout the experimental period. The change trends of the soluble sugar and soluble protein contents may have occurred because the temperature in the plain area is lower than that in the plateau area. In this experiment, the concentrations of the two kinds of active oxygen, $\mathrm{H}_{2} \mathrm{O}_{2}$ and $\mathrm{O}_{2}{ }^{-}$, increased when the altitudinal environmental conditions changed, but the change was not significant. The content of SOD increased due to the altitude change, while the contents of POD and GSH gradually returned to the control levels. The results showed that light intensity had little effect on the production of reactive oxygen species.

\section{Functional analysis of genes in common vetch related to the altitude change response}

The LHC protein is the core protein involved in photosynthesis in eukaryotes; it is composed of the LHC family and several families related to plant protection ${ }^{26}$. The lack of chlorophyll b resulted in a significant decrease in Ihcb1, Lhcb6 and Ihca1 in LHC subunits ${ }^{27,28}$. ChIH is a porphyrin-binding subunit and a key enzyme in chlorophyll synthesis ${ }^{29}$. Mg-chelatase makes protoporphyrin IX participate in chlorophyll biosynthesis, and its activity decreases with decreasing $\mathrm{ChlH}$ transcription levels ${ }^{30}$. In the 
study of the chlorophyll synthesis pathway in this experiment, chlh and other genes involved in chlorophyll synthesis (HemA, HemC, HemE), magnesium chelatase $\mathrm{H}$ subunit, magnesium protoporphyrin oxymethyltransferase and other genes were downregulated. This result is consistent with the decrease in the chlorophyll content. Overall, these results indicate that LHC family genes may be involved in chlorophyll protection under low light-intensity stress in the plain altitude environment. LHC plays a role in maintaining normal metabolic activity under low-light conditions (Fig. 8, Fig. 11).

It has been reported that the content of the Cyt- $\mathrm{b}_{6} / \mathrm{f}$ complex in Arabidopsis thaliana is positively correlated with an increase in the light intensity for constant growth, that is, high light $>$ normal light $>$ low light ${ }^{31}$. The decrease in the Cyt- $\mathrm{b}_{6} / \mathrm{f}$ complex reduces the electron transport rate of PSII, which leads to a decrease in the $\mathrm{CO}_{2}$ assimilation rate ${ }^{32}$. The results show that $\mathrm{Cyt}_{\mathrm{t}} \mathrm{b}_{6} / \mathrm{f}$ has a high level of control over the electronic transmission rate of Setaria viridis in $\mathrm{C} 4$ plants ${ }^{33}$, and high Cyt- $\mathrm{b}_{6} / \mathrm{f}$ abundance in $\mathrm{C} 4$ plants may lead to a high $\mathrm{CO}_{2}$ assimilation rate and high yield ${ }^{34}$. This is consistent with the results of this experiment. The expression of $C y t-b_{6} / f$ related genes was downregulated after the seedlings were transported from high altitude to low altitude. As a result, the rate of $\mathrm{CO}_{2}$ assimilation and the photosynthetic capacity of the seedlings decreased. In pea leaf experiments, it was suggested that the activation of FD-NADP ${ }^{+}$reductase and the increase in electron flow between PSII and PSI could lead to the acceleration of NADP + reduction by FD ${ }^{35}$. ATPase plays a key role in the coregulation of light and dark reactions in photosynthesis. Experiments have suggested that photosynthesis can be controlled by regulating ATPase in tobacco under the conditions of high $\mathrm{CO}_{2}$ and low $\mathrm{O}_{2}{ }^{36}$. In this experiment, NADP + reductase and ATP synthase were downregulated. The results showed that photosynthesis in common vetch was inhibited in the low-altitude plain environment. This may have been due to the low light intensity in the plain environment or the low $\mathrm{CO}_{2}$ concentration in the air, which could have inhibited the production of photosynthesis and chlorophyll (Fig. 8, Fig. 11).

Ribulose 1,5-diphosphate carboxylase/oxygenase (Rubisco; EC 4.1.1.39) fixes carbon dioxide in the atmosphere to participate in the Calvin cycle of photosynthesis. The regeneration of RuBP is limited by Rubisco at high $\mathrm{CO} 2$ concentrations, which inhibits plant photosynthesis ${ }^{37}$. It has been confirmed in experiments that Rubisco expression in sunflower (Helianthus annuus L.) decreases under shading ${ }^{38}$. Rubisco is downregulated at high temperatures in rice ${ }^{39}$. Common vetch is a C4 plant of the NAD-ME type, which uses aspartic acid as the main transport metabolite. Aspartic acid is converted to malic acid by reductive deamination ${ }^{40}$. It has been confirmed that the Rubisco content of sunflower (Helianthus annuus L.) decreased under shading ${ }^{41}$. In this experiment, compared with the control group, 19 genes in $\mathrm{J} 3$ and $\mathrm{J} 7$ involved in carbon fixation by photosynthetic organisms were differentially expressed. The genes encoding Rubisco, phosphoglycerate kinase, glyceraldehyde-3-phosphate dehydrogenase and many enzymes involved in ribose-1,5-diphosphate (RuBP) regeneration were downregulated. (Fig. 8, Fig. 11).

\section{Conclusions}


In this study, we observed the physiological and transcriptome changes of common vetch in Plateau and plain environment, in order to reveal the adaptation mechanism of plateau plants to plain environment. The changes of leaf tissue structure and physiological indexes showed that there was an obvious "intoxicated oxygen" reaction on the first day after transporting to the plain. Further transcriptome analysis showed that there were significant changes in genes related to KEGG pathway. The results showed that the inadaptability of common vetch to plain environment was mainly affected by different light intensity.

\section{Declarations}

\section{Funding}

This work was supported by National Natural Science Foundation of China (31872998).

\section{Competing interests}

The authors declare no competing interests.

\section{Statement}

The use of plant parts in present study compiles with international, national guidelines. Seeds of common vetch (Vicia sativa L.), variety XiMu324, obtained from the Qinghai Academy of Animal Husbandry and Veterinary Sciences. The author has obtained the permission to use them.

\section{References}

1. Li, G. et al. Grazing alters the phenology of alpine steppe by changing the surface physical environment on the northeast Qinghai-Tibet Plateau, China. Journal of Environmental Management 248(Oct.15):109257.1-109257.7(2019).

2. Senica, M. et al. The higher the better Differences in phenolics and cyanogenic glycosides in Sambucus nigra leaves, flowers and berries from different altitudes. Journal of the ence of Food \& Agriculture, 97 (8), 2623-2632 (2017).

3. Pepin, N. et al. Elevation-dependent warming in mountain regions of the world.Nature Climate Change424-430(2015).

4. Piao, S. et al. The impacts of climate change on water resources and agriculture in China. Nature, 467 (7311), 43-51 (2010).

5. Ke, H. et al. The Influences of Climate Change and Human Activities on Vegetation Dynamics in the Qinghai-Tibet Plateau. Remote Sensing, 8 (10), 876 (2016).

6. Qiao, Q. et al. Transcriptome sequencing of Crucihimalaya himalaica (Brassicaceae) reveals how Arabidopsis close relative adapt to the Qinghai-Tibet Plateau.Nature(2016). 
7. Yang, S. et al. Characterization of the Tibet plateau Jerusalem artichoke (Helianthus tuberosus L.) transcriptome by de novo assembly to discover genes associated with fructan synthesis andSSR analysis156(1) (2019).

8. Zhou, D. et al. De novo sequencing transcriptome of endemic Gentiana straminea (Gentianaceae) to identify genes involved in the biosynthesis of active ingredients.Gene575(1) (2016).

9. Qiao, Q. et al. Transcriptome sequencing of Crucihimalaya himalaica (Brassicaceae) reveals how Arabidopsis close relative adapt to the Qinghai-Tibet Plateau. Rep, 6, 21729 (2016).

10. Pietrzykowska, M. et al. The Light-Harvesting Chlorophyll a/b Binding Proteins Lhcb1 and Lhcb2 Play Complementary Roles during State Transitions in Arabidopsis., 26, 3646-3660 (2014).

11. Huie, L. et al. De Novo Assembly and Discovery of Genes That Are Involved in Drought Tolerance in Tibetan Sophora moorcroftiana. Plos One, 10 (1), e111054 (2015).

12. Fu, J. et al. De novo transcriptome sequencing and gene expression profiling of Elymus nutans under cold stress. BMC Genom, 17 (1), 870 (2016).

13. Tae-Sung, K. et al. Transcriptome Analysis of Two Vicia sativa Subspecies: Mining Molecular Markers to Enhance Genomic Resources for Vetch Improvement. Genes, 6 (4), 1164-1182 (2015).

14. Li, R. et al. Effects of Cultivar and Maternal Environment on Seed Quality in Vicia sativa. Frontiers in Plant Science, 8, 1411-1419 (2017).

15. Aouida, M. et al. Polymorphisms in cyanogenic glucoside and cyano-amino acid content in natural accessions of common vetch (Vicia sativa L.) and selection for improved agronomic performance.Plant Breeding(2019).

16. Makkar, H. P. S. et al. Seaweeds for livestock diets: A review. Animal Feed ence \& Technology, 212, 117 (2016).

17. Lin, K. H. et al. Physiological Characteristics of Photosynthesis in Yellow-Green, Green and DarkGreen Chinese Kale (Brassica oleracea L. var. alboglabra Musil.) under Varying Light Intensities. Plants(2020).

18. Wang, C. L. et al. Physiological characteristics, dry matter, and active component accumulation patterns of Changium smyrnioides in response to a light intensity gradient[J]. Pharm. Biol, 55 (1), 581 (2017).

19. Meng, Z. J. et al. Subhigh Temperature and High Light Intensity Induced Irreversible Inhibition on Photosynthesis System of Tomato Plant (Solanum lycopersicum L.). FRONT PLANT, 8, 365-381 (2017).

20. Terashima, I. \& Hanba, Y. T. Danny Tholen and ülo Niinemets. Leaf Functional Anatomy in Relation to Photosynthesis[J]. Plant Physiol, 155 (01), 108-116 (2011).

21. Fan, Y. et al. Soybean (Glycine max L. Merr.) seedlings response to shading: leaf structure, photosynthesis and proteomic analysis.BMC Plant Biology19(1) (2019).

22. Jumrani, K. \& Bhatia, V. S. Influence of different light intensities on specific leaf weight, stomatal density photosynthesis and seed yield in soybean(2020). 
23. Zhang, Z. et al. The effect of shading on morphological and physiological indexes of five legume forages species. Pratacultural Science, 28 (7), 1296-1300 (2011).

24. Zhang, C. X. \& Hu, T. M. Effects of low temperature and weak light on physiological traits of alfafa's root crown. Pratacultural Science, 27 (5), 72-75 (2010).

25. Jin, L. Z. et al. Effects of High Temperature Stress on Physiological and Biochemical Traits of Soybeans with Different Heat Tolerance. Soybean Science, 38 (1), 63-71 (2019).

26. Engelken, J., Brinkmann, H. \& Adamska, I. Taxonomic distribution and origins of the extended LHC (light-harvesting complex) antenna protein superfamily. BMC Evol Biol, 10, 233 (2010).

27. Sato, R., Ito, H. \& Tanaka, A. Chlorophyll b degradation by chlorophyll b reductase under high-light conditions. Photosynth. Res, 126 (2), 249-259 (2015).

28. Tyutereva, E. V. et al. The absence of chlorophyll b affects lateral mobility of photosynthetic complexes and lipids in grana membranes of Arabidopsis and barley chlorina mutants.Photosynthesis Research(2017).

29. Xiaoqin, L., Yue, L. \& Shangwei, Z. Interplay between Light and Plant Hormones in the Control of Arabidopsis Seedling Chlorophyll Biosynthesis. Frontiers in Plant ence, 8, 1433 (2017).

30. Luo, S. et al. N-terminus plus linker domain of Mg-chelatase D subunit is essential for Mg-chelatase activity in Oryza sativa [J]. Biochemical \& Biophysical Research Communications, 497 (2), 749 (2018).

31. Alsharafa, K. Acclimation of normal or low light grown Arabidopsis thaliana to high light(2012).

32. Kubo, S. et al. Cyclic electron flow around PSI functions in the photoinhibited rice leaves. Soil ence \& Plant Nutrition, 57 (1), 105-113 (2011).

33. Ermakova, M. et al. Overexpression of the Rieske FeS protein of the Cytochrome b6/f complex increases C4 photosynthesis in Setaria viridis. Communications Biology, 2 (1), 1-12 (2019).

34. Yin, X. \& Struik, P. C. Mathematical review of the energy transduction stoichiometries of C4 leaf photosynthesis under limiting light. Plant Cell \& Environment, 35 (7), 1299-1312 (2012).

35. Masayoshi, S. et al. Gymnosperms have increased capacity for electron leakage to oxygen (Mehler and PTOX reactions) in photosynthesis compared with angiosperms.Plant \& Cell Physiology(7):1152-1163(2013).

36. Rott Markus, Martins Nádia, F. et al. ATP synthase repression in tobacco restricts photosynthetic electron transport, $\mathrm{CO}_{2}$ assimilation, and plant growth by overacidification of the thylakoid lumen.The Plant cell23(1) (2011).

37. Joanna, C. et al. A non-radioactive method for measuring Rubisco activase activity in the presence of variable ATP: ADP ratios, including modifications for measuring the activity and activation state of Rubisco[J]. Photosynth. Res, 119 (3), 355-365 (2014).

38. Mitra, S. \& Srivastava, G. C. Photosynthesis as Influenced by Assimilate Level in Sunflower (Helianthus annuus L.). Journal of Agronomy and Crop Science, 171 (1), 20-25 (2010). 
39. Perdomo, J. A. et al. Rubisco and Rubisco Activase Play an Important Role in the Biochemical Limitations of Photosynthesis in Rice, Wheat, and Maize under High Temperature and Water Deficit. Frontiers in Plant Science, 8, 490 (2017).

40. Xiaolan, R. \& Dixon, R. A. The Differences between NAD-ME and NADP-ME Subtypes of C4 Photosynthesis: More than Decarboxylating Enzymes.Frontiers in Plant Science7 (2016).

41. Mitra, S. \& Srivastava, G. C. Photosynthesis as Influenced by Assimilate Level in Sunflower (Helianthus annuus L.). Journal of Agronomy and Crop Science, 171 (1), 20-25 (2010).

\section{Figures}

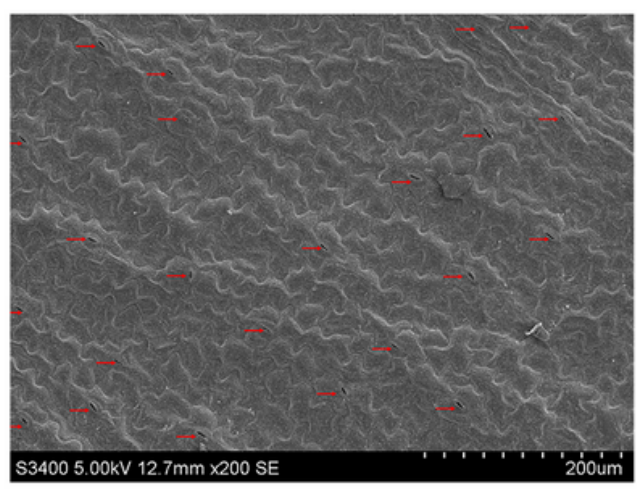

a

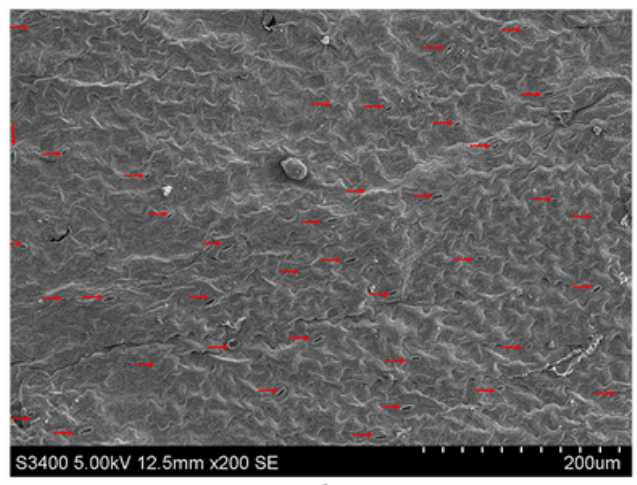

d

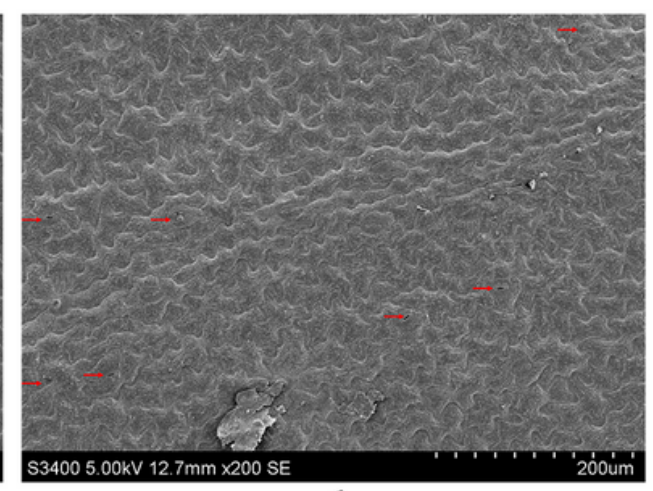

b

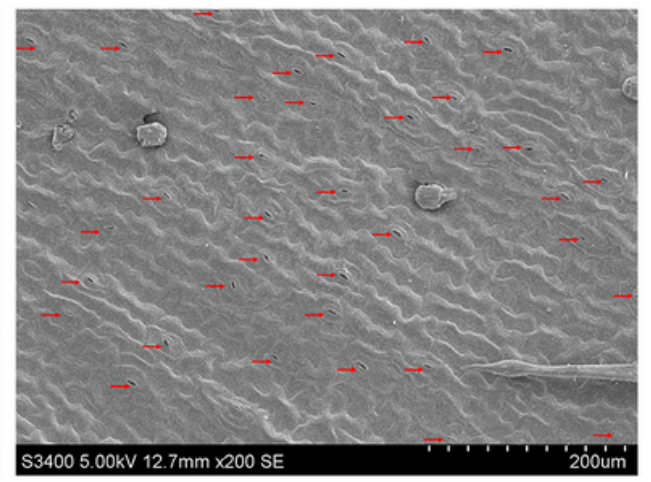

e

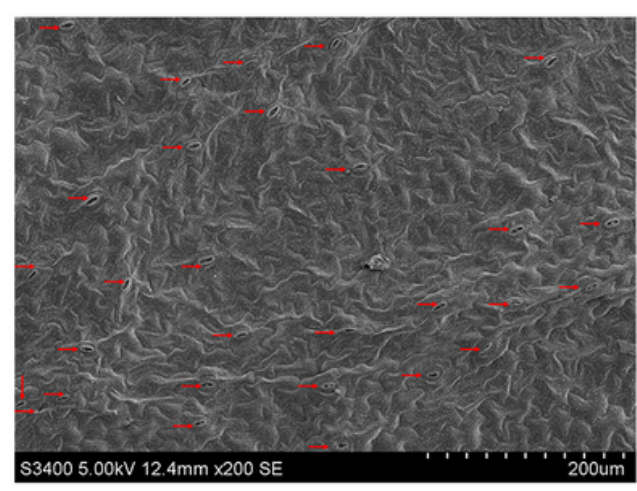

C

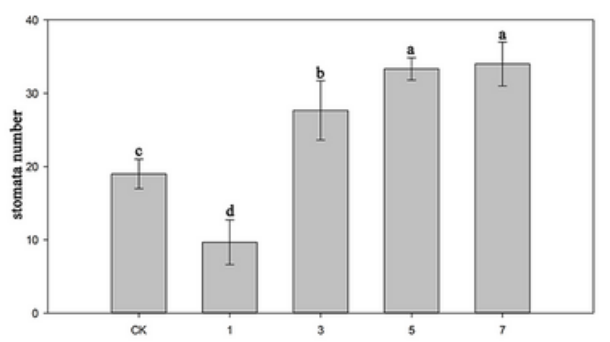

f

\section{Figure 1}

Number of stomata on common vetch leaves under plain conditions and control conditions. Images of the stomata: a control group, b the first day (J1), c the third day (J3), d the fifth day (J5), e the seventh day (J7) and $f$ the number of stomata. Significant differences between the plain condition and the control condition are indicated by different lowercase letters $(p<0.05)$ 


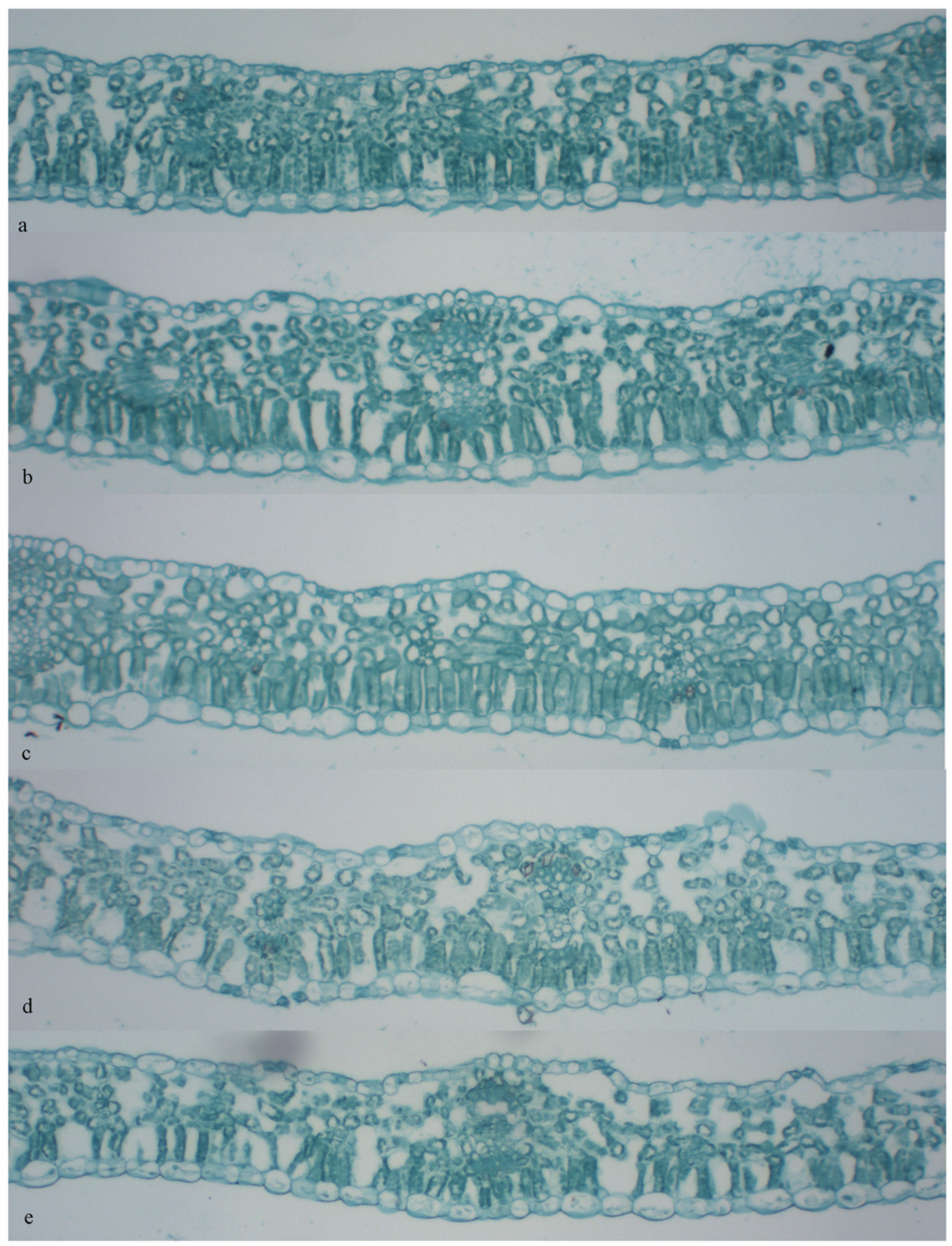

\section{Figure 2}

Anatomical structure of common vetch leaves. a Control group, b the first day (J1), c the third day (J3), d the fifth day (J5), e the seventh day. 

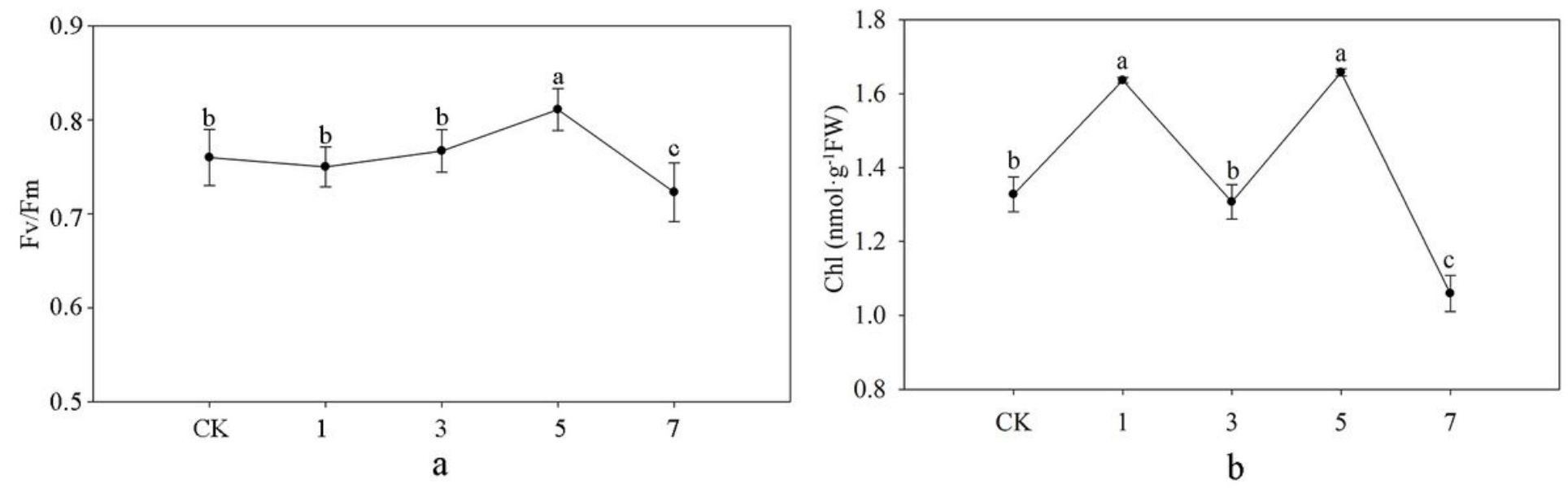

Figure 3

Changes in chlorophyll content and chlorophyll fluorescence in leaves. a Chlorophyll content and b chlorophyll fluorescence data were collected for the control condition and on 4 different days (days 1, 3 , 5 , and 7).
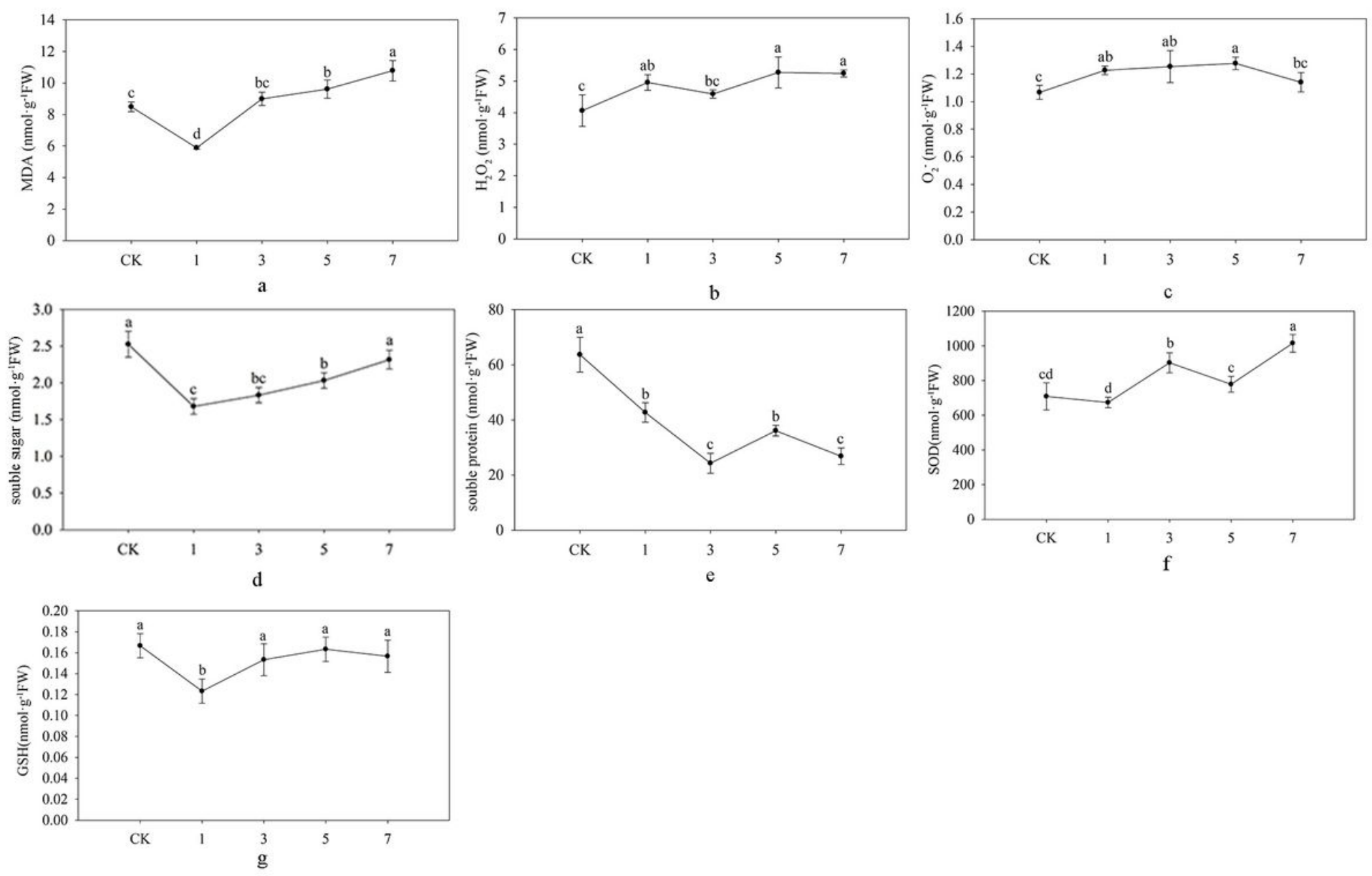

Figure 4 
Physiological changes in common vetch leaves under plain conditions. The contents of MDA a, H2O2 b, $\mathrm{O} 2$ - c, soluble sugar d, soluble protein e, SOD $\mathrm{f}$ and GSH g were determined. Three repetitions were used in the analysis, and significant differences between the plain condition and control condition are indicated by different lowercase letters $(p<0.05)$.
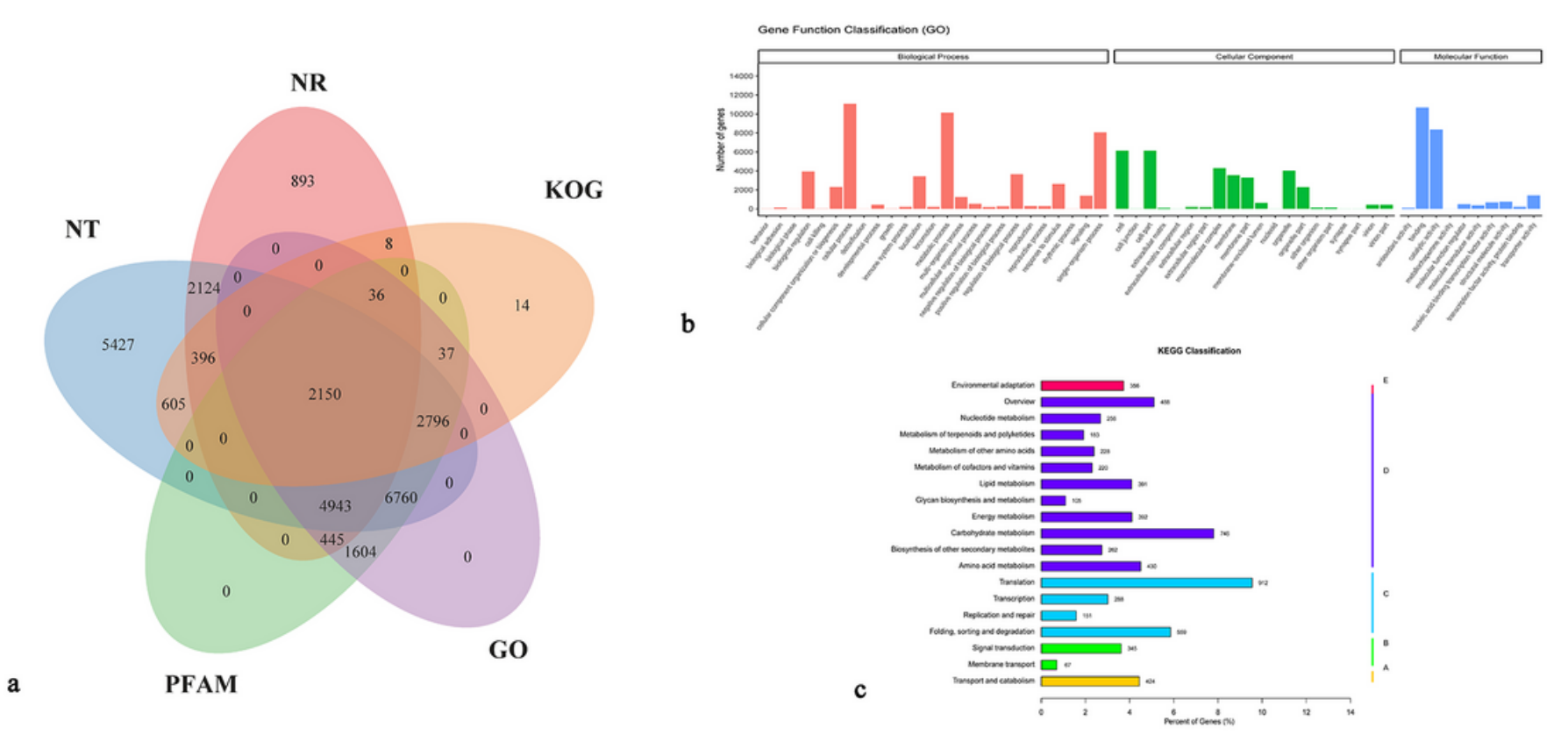

\section{Figure 5}

Annotation and functional classification of unigenes. a Venn diagram of unigenes functionally annotated for leaves. b GO annotation. The x-axis is the GO classification of gene function, and the $y$-axis is the number of genes in the category. c KEGG classification. The x-axis is the number of genes in the interpretation, and the $y$-axis is the classification of gene function
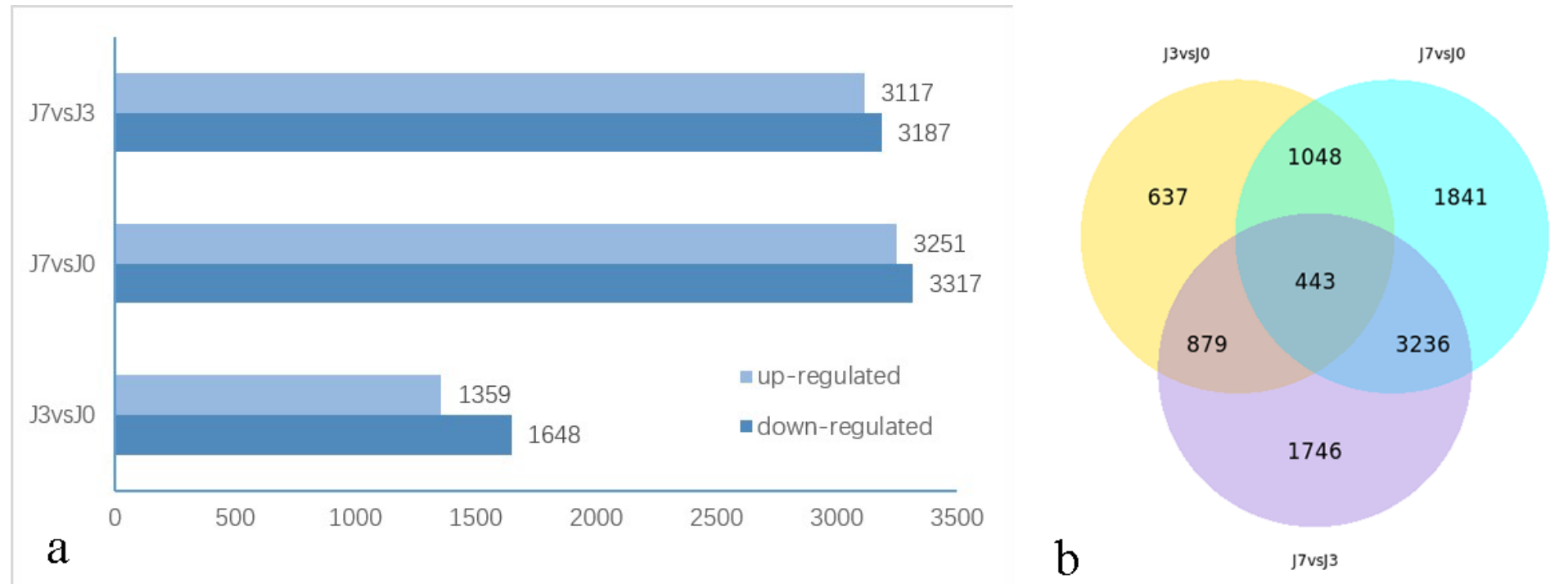

Figure 6 
DEGs between the J3 and J7 plain samples and the control samples. a Summary of the number of DEGs between the plain and control conditions. $b$ Venn diagram indicating the DEGs identified by J3 vs J0, J7 vs $\mathrm{J} 0$ and $\mathrm{J} 3$ vs $\mathrm{J} 7$ comparisons
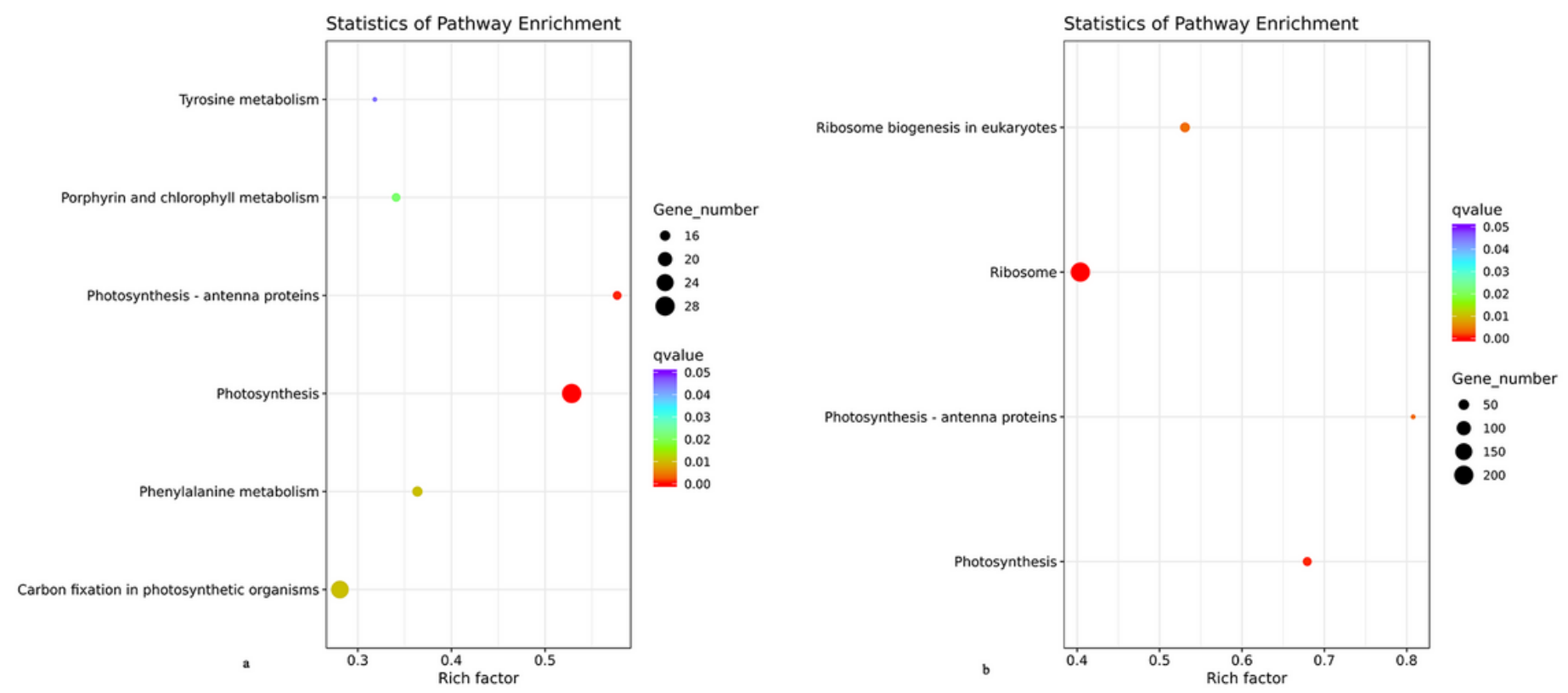

\section{Figure 7}

Enriched KEGG pathways of the annotated DEGs. The left Y-axis indicates the KEGG pathway. The X-axis indicates the Rich factor. A high q value is represented by blue. a J3vsJ0, b J7vs J0. 

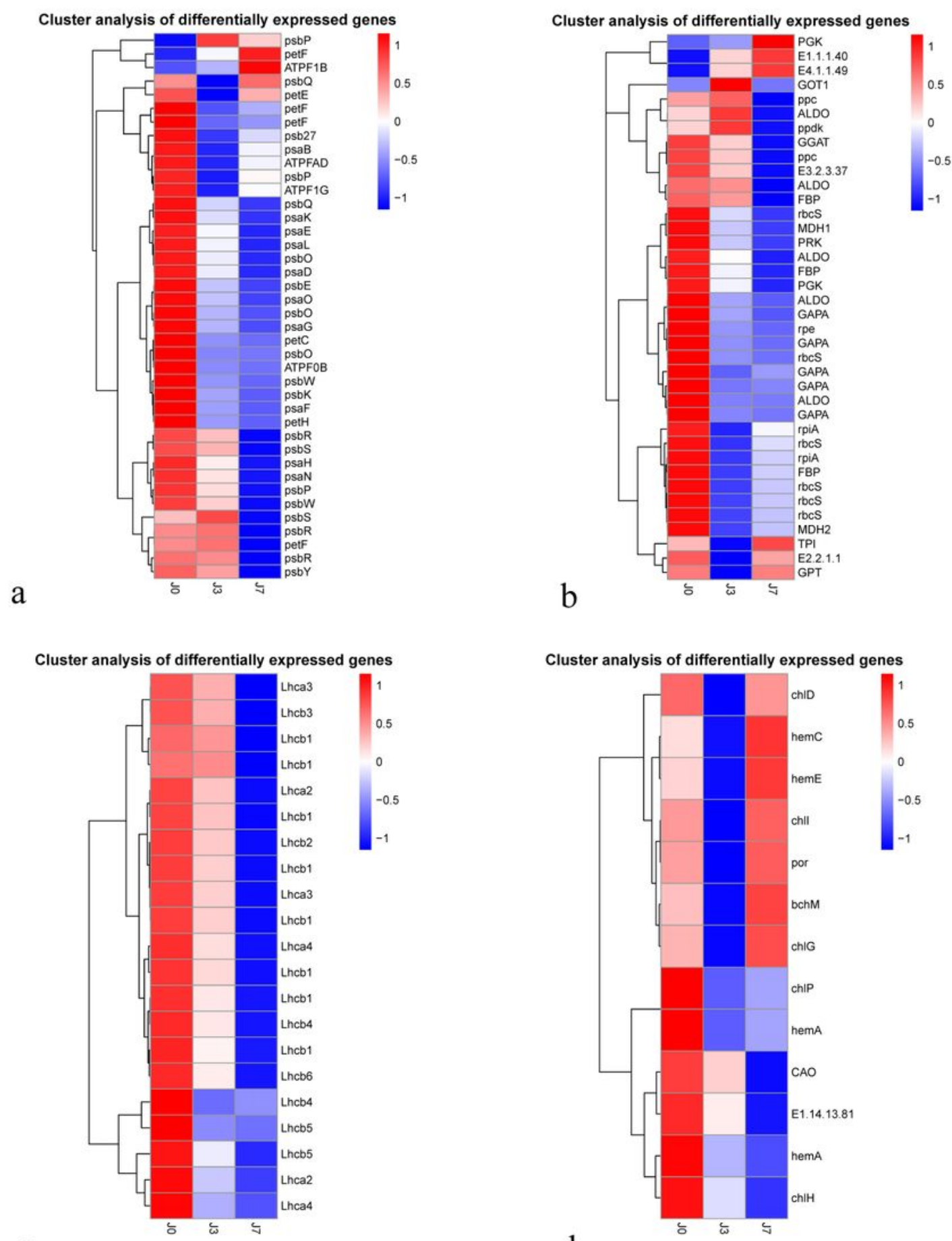

C

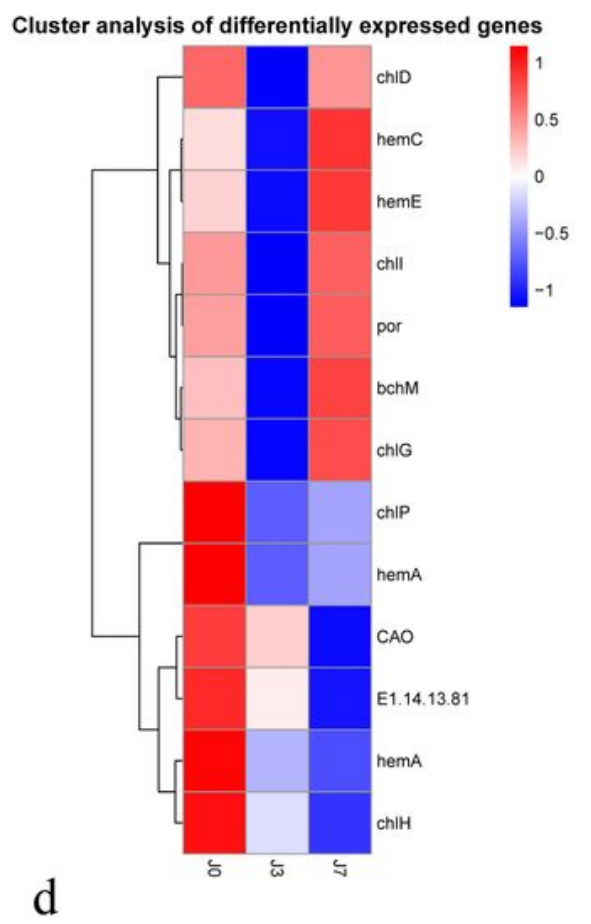

\section{Figure 8}

Heatmap of hub genes in the green module. a photosynthesis, b Carbon fixation in photosynthetic organisms, c photosynthesis - antenna proteins, d porphyrin and chlorophyll metabolism. 


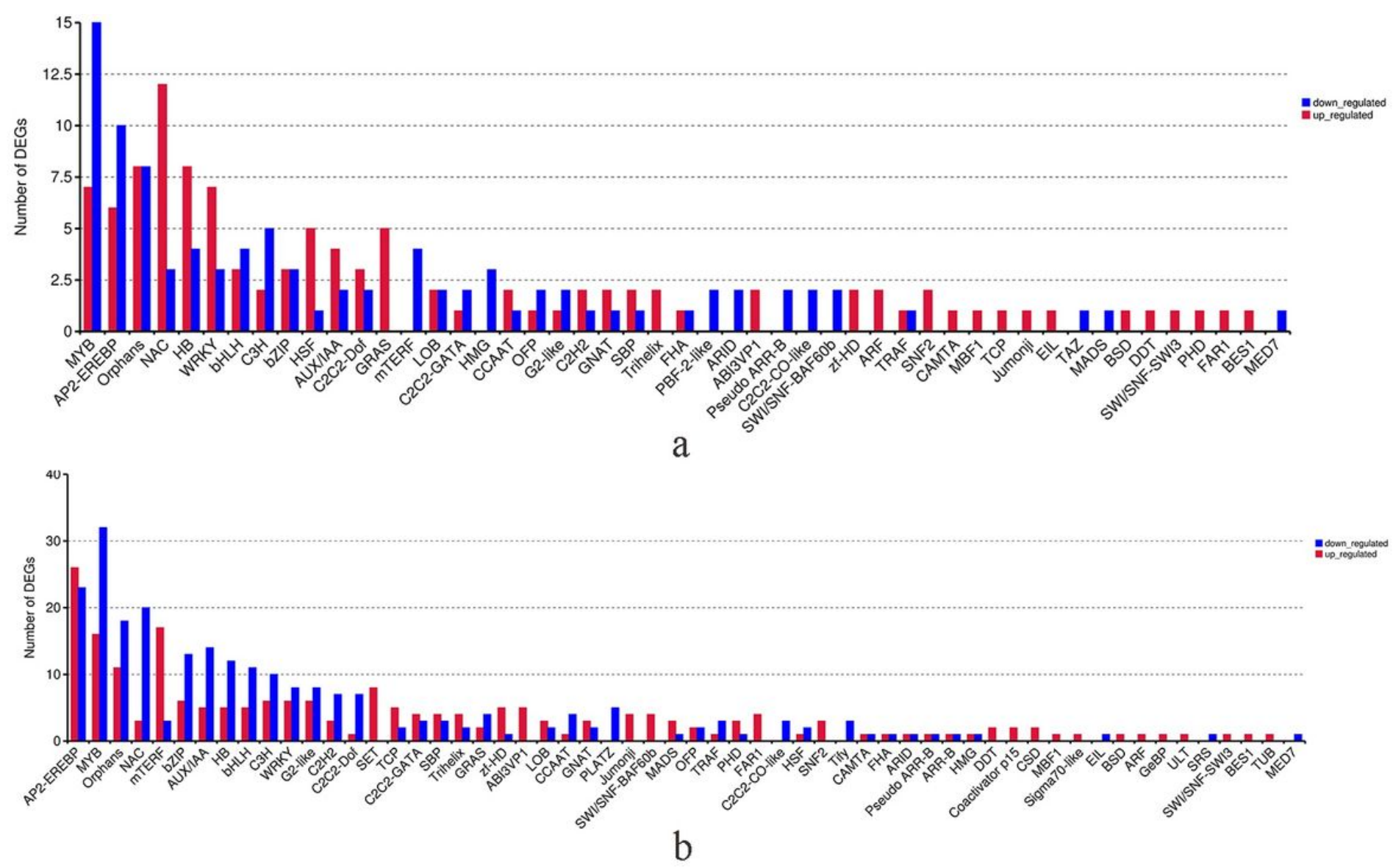

Figure 9

Statistics on the number of differential transcription factors. The $x$-axis is the name of transcription factor, and the $y$-axis is the number of genes. a J3 vs J0, b J7 vs J0. 

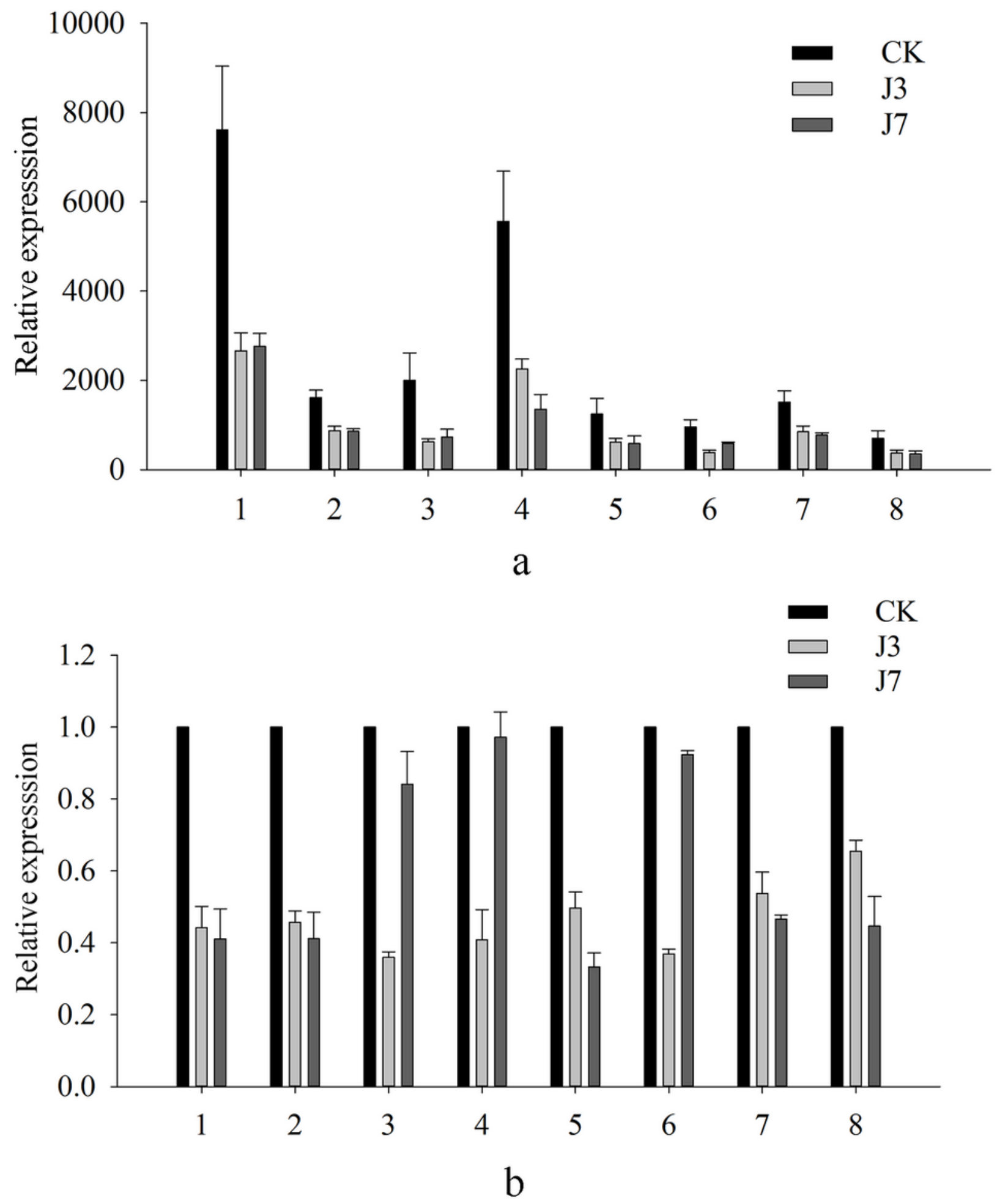

Figure 10

Quantitative RT-PCR confirmation of certain downregulated unigenes. The y-axis represents the relative quantification (RQ) for the $\mathrm{J} 3$ vs $\mathrm{J} 0$ and $\mathrm{J} 7 \mathrm{vs} \mathrm{J} 0$ treatments, and the error bars indicate the standard deviations of three replicates. 1 (Cluster-7705.13598), 2 (Cluster-7705.13381), 3 (Cluster-7705.13418), 4 (Cluster-7705.13113), 5 (Cluster-7705.13726), 6 (Cluster-7705.13438), 7 (Cluster-7705.13612), and 8 (Cluster-7705.14151). The three columns are J0, J3 and J7. 

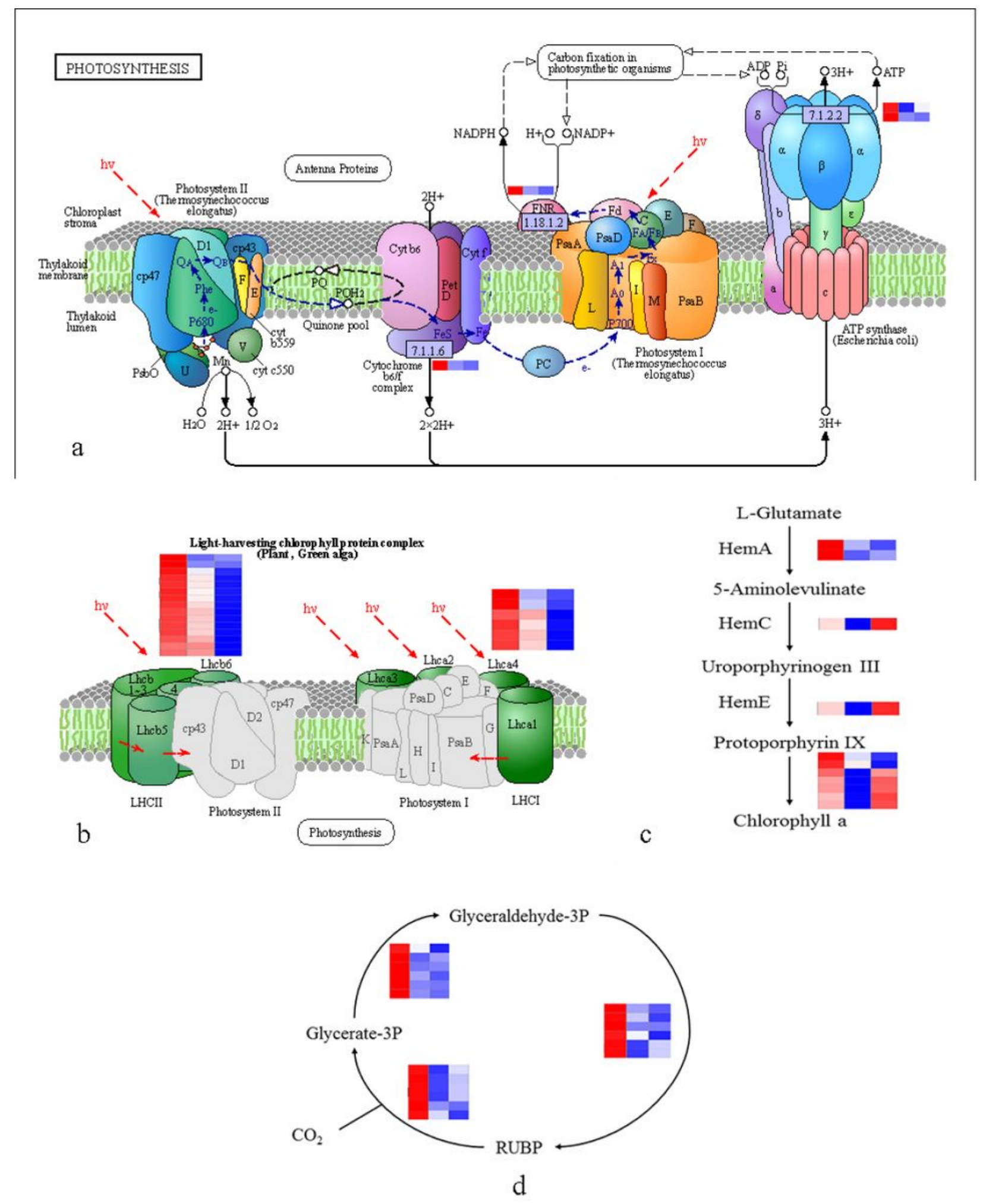

\section{Figure 11}

Responsive progression of gene expression under J0 vs J3 and J7 vs J0. a Photosynthesis, b photosynthesis - antenna proteins, c porphyrin and chlorophyll metabolism, $\mathrm{d}$ carbon fixation in photosynthetic organisms.

\section{Supplementary Files}


This is a list of supplementary files associated with this preprint. Click to download.

- Supplementaryfile1TableS1TableS2FigureS1.docx

- Supplementaryfile2TotalGOenrichment.xls

- Supplementaryfile3KEGGPathwaysandGenesEnriched.xls

- Supplementaryfile4J3vsJ0.DEGKEGGpathwayenrichment.xls

- Supplementaryfile5J7vsJ0.DEGKEGGpathwayenrichment.xls

- Supplementaryfile6J3vsJ0TFDEGdetailedstat.xls

- Supplementaryfile7J7vsJ0TFDEGdetailedstat.xls 